\title{
Une application de la cartographie de potentiel à la caractérisation des matériaux: étalement de charge et effets anormaux sur des films de Polyéthylène Téréphtalate
}

\author{
Delphine Koch* — Philippe Molinié* \\ * Supélec, Département Energie \\ Plateau du Moulon, 91192 Gif-sur-Yvette Cedex \\ delphine.koch@supelec.fr \\ philippe.molinie@supelec.fr
}

\begin{abstract}
RÉSUMÉ. Nous présentons des mesures de cartographie de potentiel de surface effectuées dans le cadre du GdR $M E^{2} M S$ du CNRS, sur des films de polyéthylène téréphtalate. Elles mettent en évidence une migration latérale de la charge, une injection dans certains cas, ainsi que des effets anormaux de remontée du potentiel après dépôt de charges. Diverses modélisations ont été développées mais elles ne semblent pas suffisantes pour expliquer les effets observés. Des effets piézoélectriques ou des forces de tension superficielle pourraient être invoqués pour rendre compte d'un déplacement de la charge contre le champ électrique.
\end{abstract}

ABSTRACT. We present here surface potential measurements on PET films, which show evidence for lateral charge spreading, charge injection, and anomalous effects such as a voltage rise occurring after charge deposit. Several models have been developed, but they do not fully account for the observed results. Piezoelectric effects or surface tension forces might also be invoked to explain charge spreading against the electrical field.

MOTS-CLÉS : isolation électrique, films polymères, polyéthylène téréphtalate, potentiel de surface, injection de charge, pression électrostatique.

KEYWORDS: electrical insulation, polymer films, polyethylene terephthalate, surface potential, charge injection, electrostatic pressure. 


\section{Introduction}

Les sondes électrostatiques permettant, sans contact, la mesure du potentiel d'une surface isolante sont utilisées depuis les années 1970, pour la caractérisation de l'écoulement de la charge sur les matériaux isolants. Elles sont d'emploi très simple, et sont habituellement utilisées pour mesurer en un point de la surface d'un isolant le «déclin de potentiel » qui suit la charge (par contact, ou par décharge corona, par exemple) d'une partie de cette surface. L'utilisation de ce type de sonde pour produire des images électrostatiques des surfaces par cartographie est moins répandue, mais des travaux précédents (voir par exemple Molinié 2004) ont montré l'utilité de cette technique pour la détection de défauts sur un revêtement.

Nous avons effectué des mesures de potentiel de surface sur des films de Polyéthylène Téréphtalate (PET), dans le cadre de l'opération Matériaux Diélectriques du GdR CNRS $\mathrm{ME}^{2} \mathrm{MS}$ «Synergie des contraintes et durabilité des isolants organiques », pour laquelle le PET a été choisi comme matériau de référence, ce qui a permis de caractériser dans plusieurs laboratoires, par diverses techniques, des films issus du même fournisseur et des mêmes plaques ou bobines, pour un nombre limité d'épaisseurs différentes. Divers résultats de ces travaux communs ont déjà été publiés (Agnel 2003, Jomni 2004) et d'autres font l'objet de publications à cette conférence (Notingher 2005). L'intérêt porté sur la caractérisation de ce matériau organique est justifié par les nombreuses applications actuelles (Füzesséry) concernant l'isolation électrique de composants du génie électrique (condensateurs, transformateurs,...), mais également dans de vastes domaines de l'audiovisuel (bandes magnétiques). Le travail que nous présentons ici, en marge de ces investigations communes sur le PET, est centré sur des observations répétées d'effets que l'on peut qualifier «d'anormaux» lors de nos mesures de potentiel de surface, qui sont loin d'être entièrement élucidés mais pour lesquels nous présentons diverses interprétations.

\section{Expériences}

\subsection{Dispositif expérimental}

Nous utilisons un banc de mesure de potentiel de surface, comprenant un poste de charge par décharge couronne, et un poste de mesure de potentiel.

La face métallisée du film isolant est posée sur un porte-échantillon relié à la masse, qui est déplacé par un vérin entre les deux postes. Pendant la charge, le potentiel de surface est régulé par une grille métallique entre la source corona et l'échantillon $\left(\mathrm{d}_{\text {grille/film }} \sim 0,5 \mathrm{~cm}\right)$, ce qui permet de charger la surface au même potentiel que la grille. Nous utilisons un dispositif pointe-grille circulaire $\left(\mathrm{d}_{\text {pointe/grille }} \sim 1 \mathrm{~cm}\right)$, permettant de déposer un disque de charge sur la surface, d'un diamètre (environ $17 \mathrm{~mm}$ ) nettement inférieur aux dimensions de l'échantillon. Le 
temps de charge retenu est faible, de l'ordre d'une seconde pour ne pas altérer l'état physicochimique de la surface du matériau.

La mesure du potentiel de surface est réalisée par un voltmètre électrostatique basé sur une classique sonde sans contact Monroe (Menguy), à condensateur vibrant, asservi au potentiel de la surface, afin d'annuler le champ électrique dans l'espace entre celle-ci et la surface à mesurer. Cette sonde est mobile sur un axe, le porte-échantillon étant mobile sur l'axe perpendiculaire, ce qui permet une cartographie en deux dimensions du potentiel (dont la durée est de l'ordre d'une trentaine de minutes). La résolution latérale de la sonde est de l'ordre de 0,5 mm. Le temps de réponse est de moins de $80 \mu \mathrm{s}$, et la précision de mesure est de $0,1 \%$ pour un espacement entre la surface et la sonde de $1,5 \mathrm{~mm}$.

\subsection{Paramètres des essais}

Les expériences ont été menées sur des films de PET semi-cristallin métallisés sur une face à l'or par évaporation. Nous présentons des résultats obtenus sur des échantillons d'épaisseur $23 \mu \mathrm{m}$, mais également quelques mesures réalisées sur un échantillon de $100 \mu \mathrm{m}$ (lorsque rien n'est précisé, les mesures présentées concernent les films de $23 \mu \mathrm{m})$.

Nous avons effectué après une charge initiale de $+2000 \mathrm{~V}$, des cartographies successives du potentiel, dont les échelles sont représentées en volts. Leurs dimensions sont de $45 / 45 \mathrm{~mm}$ avec une mesure tous les $0,5 \mathrm{~mm}$, excepté celle de la figure 1 , de dimension $35 / 35 \mathrm{~mm}$ avec une mesure tous les $0,35 \mathrm{~mm}$. Le champ initial moyen calculé est donc de $87 \mathrm{kV} / \mathrm{mm}$ pour le film de $23 \mu \mathrm{m}$, et de $20 \mathrm{kV} / \mathrm{mm}$ pour celui de $100 \mu \mathrm{m}$. Nous avons observé brièvement l'influence du courant de charge sur la dynamique du potentiel de surface. Les courants de charge appliqués sont entre 8 à $40 \mu \mathrm{A}(20 \mu \mathrm{A}$ par défaut). Ces mesures ont été réalisées à une température de $20^{\circ} \mathrm{C}$ et une humidité relative d'environ $50 \%$.

\subsection{Représentations dérivées}

A l'aide des relevés expérimentaux, on a effectué l'intégration du potentiel sur toute la surface des cartographies, de manière à calculer le potentiel moyen. Ce potentiel est donné figures 4 et 5 , en représentation $\left(V-V_{0}\right) / V_{0}$ en fonction du temps $\left(\mathrm{V}_{0}\right.$ étant le potentiel initial). L'intérêt d'un tel calcul, est à priori de pouvoir évaluer, dans un contexte marqué par une migration latérale de la charge, l'influence de phénomènes de volume sur le déclin de potentiel.

Nous montrons aussi des cartographies différentielles en deux dimensions, qui sont obtenues par simple différence des potentiels entre deux cartographies successives réalisées durant le déclin. Cela permet d'illustrer les zones de perte ou gain de potentiel sur la surface de l'échantillon, et donc de représenter la dynamique des charges. 


\section{Résultats}

\subsection{Evolution temporelle des cartographies de potentiel}

Nous avons observé (figure 1), l'évolution sur quinze jours, l'état de surface d'un des échantillons d'épaisseur $23 \mu \mathrm{m}$, après une succession de charges appliquées à quelques jours d'intervalle, avec une alternance de polarité.

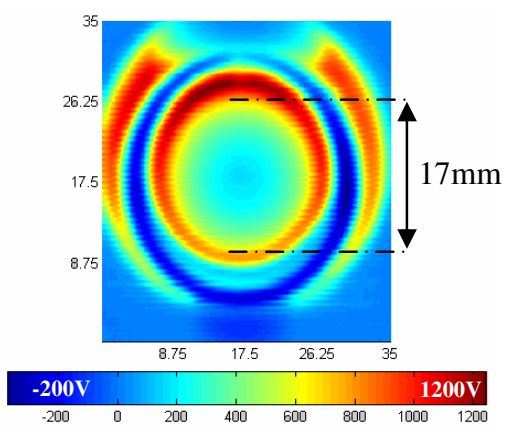

Figure 1. Cartographie de potentiel après des dépôts de charge positifs et négatifs

Nous observons des couronnes positives et négatives d'un diamètre bien supérieur à celui de la charge initiale, qui montrent un élargissement progressif de la distribution. De plus, aucune orientation ne semble être privilégiée.

Par conséquent, il nous a semblé utile d'analyser la progression de la charge dans le temps, après un dépôt de charge unique. Le potentiel étant de $+2000 \mathrm{~V}$ à l'instant $\mathrm{t}=0$, des séries de cartographies successives ont été réalisées sur des échantillons d'épaisseur $23 \mu \mathrm{m}$ pendant une longue période. Nous présentons figure 2 , un exemple de cartographie du potentiel parmi d'autres dans une de ces séries (série $\left.\mathrm{n}^{\circ} 1\right)$.

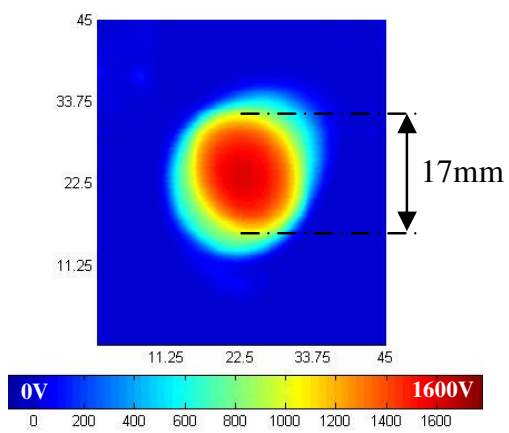

Figure 2. Cartographie du potentiel 21h après la charge 
Le dépôt initial de charge étant homogène, les séries de cartographies de potentiel mettent en évidence une migration latérale lente des charges, qui présentent une direction préférentielle, en diagonale dans le cas de cet échantillon. La figure 3 représente l'évolution du potentiel de la surface suivant cette diagonale pour trois cartographies de la série $\mathrm{n}^{\circ} 1$, dont celle de la figure 2 .

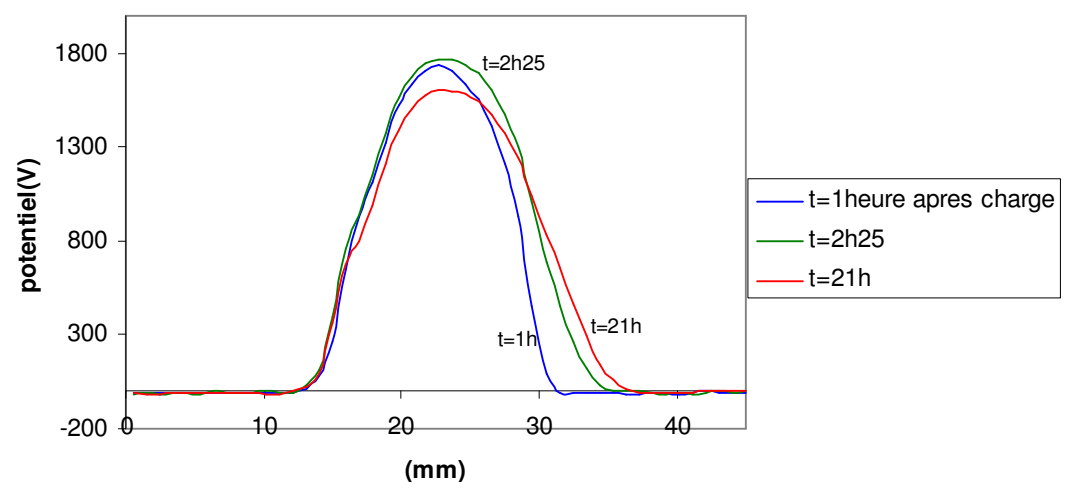

Figure 3. Evolution du potentiel de surface en fonction de la diagonale après une charge initiale $+2000 \mathrm{~V}$

Nous discernons une superposition de plusieurs phénomènes : un déplacement des charges, puis un déclin de potentiel à long terme. Soulignons que l'écoulement des charges ne s'effectue que d'un seul coté de la diagonale. Par ailleurs, ce suivi en fonction du temps du potentiel révèle une remontée initiale du potentiel de la surface, au centre de la zone chargée, de l'ordre de $40 \mathrm{~V}$, le maximum de potentiel étant observé après plusieurs heures d'évolution du potentiel.

Mentionnons également que, lors de l'essai décrit par les figures 2 et 3, une décharge surfacique a eu lieu après la cartographie réalisée $21 \mathrm{~h}$ après la charge, provoquant une diminution brutale du potentiel, suivie d'une nouvelle remontée du potentiel pendant un certain temps (voir figure 5).

\subsection{Evolution temporelle par intégration}

Nous représentons (figure 4) la valeur du potentiel moyen sur la surface, pour plusieurs séries d'essais sur quelques films différents. Nous observons des résultats très dispersés, mais on peut regrouper les mesures en deux séries, présentant ou non une remontée initiale de l'ordre de $2 \%$. La série évoquée précédemment, correspondant aux figures 2 et 3 , correspond à la courbe $n^{\circ} 1$.

Il est difficile de dire quel facteur expérimental explique la séparation de ces deux familles. Il semble que la dynamique du déclin de potentiel se trouve influencée par le régime de la décharge corona (l'augmentation du courant de 
décharge accélérant le déclin initial, sa diminution le ralentissant), mais on n'a pas trouvé de corrélation reproductible.

Par ailleurs, la remontée a également été observée, avec une amplitude comparable, sur un film de $100 \mu \mathrm{m}$.

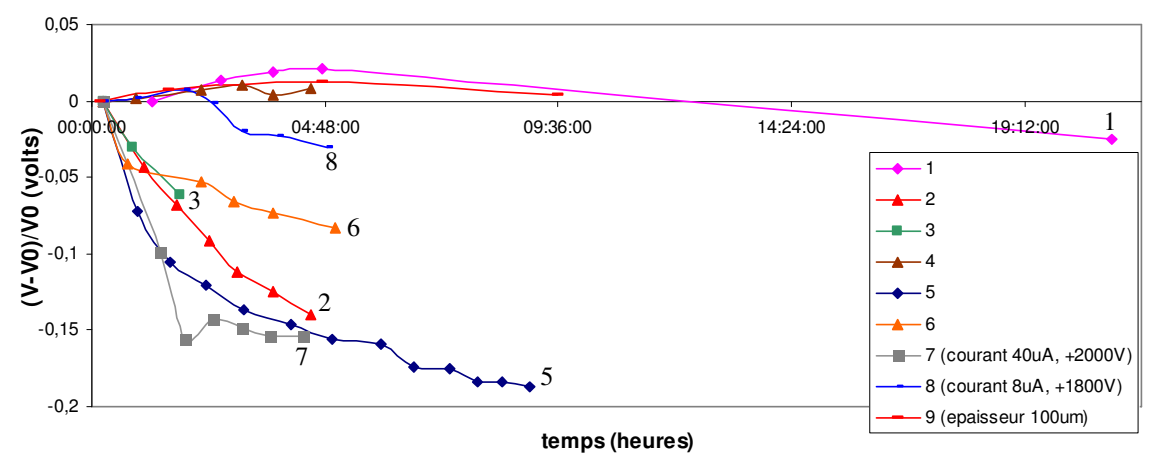

Figure 4. Evolution du potentiel moyenné sur la surface

La présence d'une décharge surfacique lors de l'essai $n^{\circ} 1$, évoquée plus haut, conduit à l'évolution de potentiel représentée ci-dessous. Cette seconde remontée semble obéir à une loi proche de la première, et peut être un indice important pour la compréhension du phénomène.

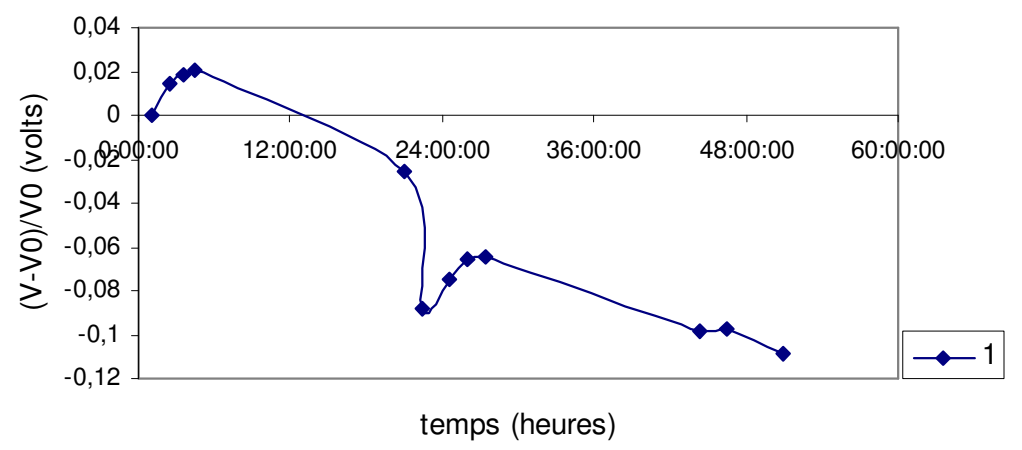

Figure 5. Potentiel moyen pour la série 1 avant et après décharge surfacique

\subsection{Evolution des cartographies différentielles}

Nous représentons figure 10 , des cartographies différentielles en référence avec la série 8 de la figure 4 , où le courant était de $8 \mu \mathrm{A}$ et où nous observions une remontée du potentiel moyen suivi d'un déclin de potentiel. Ainsi, la zone où ce situe cette remontée est visible sur les cartographies différentielles a et b. 


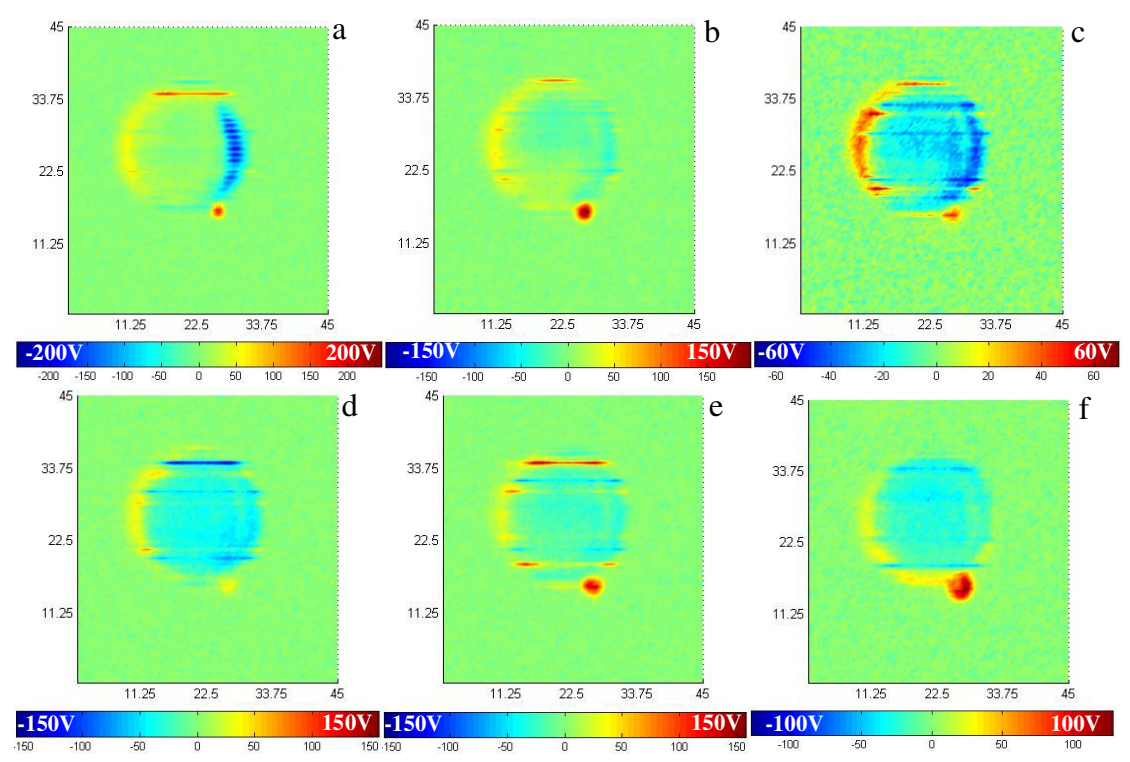

Figure 10. Cartographies différentielles, série $n^{\circ} 8,8 \mu A(a:$ entre 15 min et $1 \mathrm{~h}$, $b: 1 h$ et $2 h, c: 2 h$ et $2 h 30, d: 2 h 30$ et $3 h$, e $: 3 h$ et $4 h, f: 4 h$ et $5 h$ )

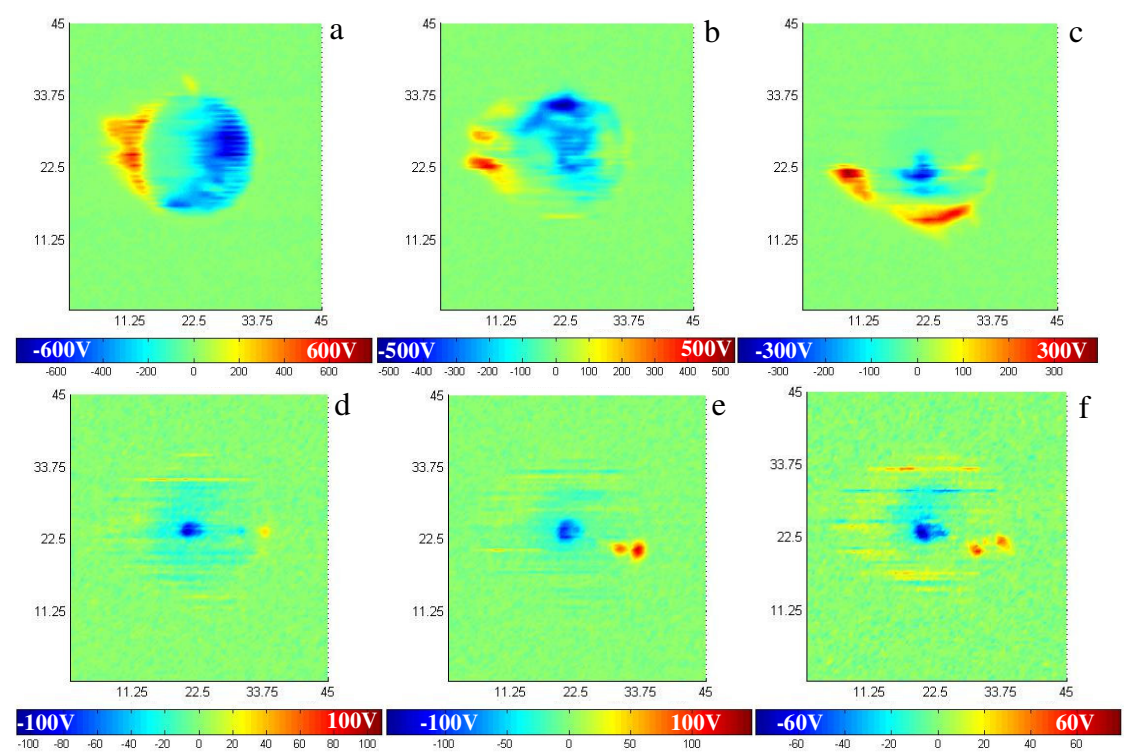

Figure 11. Cartographies différentielles, série $n^{\circ} 7,40 \mu \mathrm{A}(a$ : entre $15 \mathrm{~min}$ et $1 h 30, b: 1 h 30$ et $2 h, c: 2 h$ et $2 h 30, d: 2 h 30$ et $3 h$, e $: 3 h$ et $4 h, f: 4 h$ et $4 h 30$ ) 
La figure 11 représente également des cartographies différentielles, cette fois faisant référence à la série 7 de la figure 4 où le courant de pointe était de $40 \mu \mathrm{A}$. On note une direction préférentielle de la migration des charges sans lien avec le gradient de potentiel. De plus, nous percevons une baisse rapide du potentiel sur la zone centrale de la distribution de charge, qui était absente sur les cartographies différentielles de la figure 10. C'est dans cette zone centrale que l'augmentation du courant de la décharge affecte la cartographie

\section{Deux modèles pouvant expliquer la remontée du potentiel}

\subsection{Position du problème}

Les principaux mécanismes habituellement considérés pour expliquer le déclin de potentiel ont été recensés et analysés dans un récent article de synthèse (Molinié 2005) : ils peuvent être regroupés en trois grandes catégories, relaxation dipolaire, conduction intrinsèque, et injection de charge.

Ces différents phénomènes peuvent être invoqués pour expliquer une partie des résultats présentés ici. En revanche, l'explication de la remontée de potentiel observée dans certains cas nécessite des hypothèses particulières. La première de ces hypothèses peut entrer dans le cadre général de la dynamique du mouvement de la charge sous l'influence directe d'un champ électrique, en supposant une distribution initiale de charge particulière, les autres modèles font appel à l'intervention de divers phénomènes physico-chimiques qui puissent expliquer une migration de la charge contre le champ électrique.

\subsection{Retour de potentiel pour une distribution de charge en double couche}

Lorsqu'un matériau isolant présentant une forte charge d'espace interne répartie majoritairement près de sa surface libre est neutralisé par un dépôt de charge de polarité inverse, un «retour de potentiel » peut être dû à la remontée de la charge interne vers la surface (Coelho 1987, Molinié Alvarez 2005). Nous proposons d'examiner si un modèle de ce type, dans le cas d'une neutralisation incomplète, pourrait s'appliquer ici. Nous faisons l'hypothèse que dans certains cas, la charge initiale du film n'est pas homopolaire, mais consiste en une double couche de charges de signes opposés, la charge majoritaire, la plus importante et également celle injectée en premier (positive ici), étant accompagnée d'une charge minoritaire de signe opposée (négative), déposée sur la surface, selon la figure 12. (Nous examinerons plus loin si une telle hypothèse est réaliste).

Dans un modèle unidimensionnel, en considérant que les dimensions latérales de l'isolant (de permittivité $\varepsilon$ ) sont grandes par rapport à son épaisseur d, nous modélisons cette situation par la présence de deux plans de charge dans le volume de l'isolant, selon la figure 12. Une densité de charge $\sigma_{2}$ est présente dans le volume 
à la profondeur $\lambda$, et une densité de charge $\sigma_{1}$ de signe opposée et de plus faible valeur est à la surface de l'échantillon. Le champ électrique est supposé nul à l'extérieur de l'isolant.
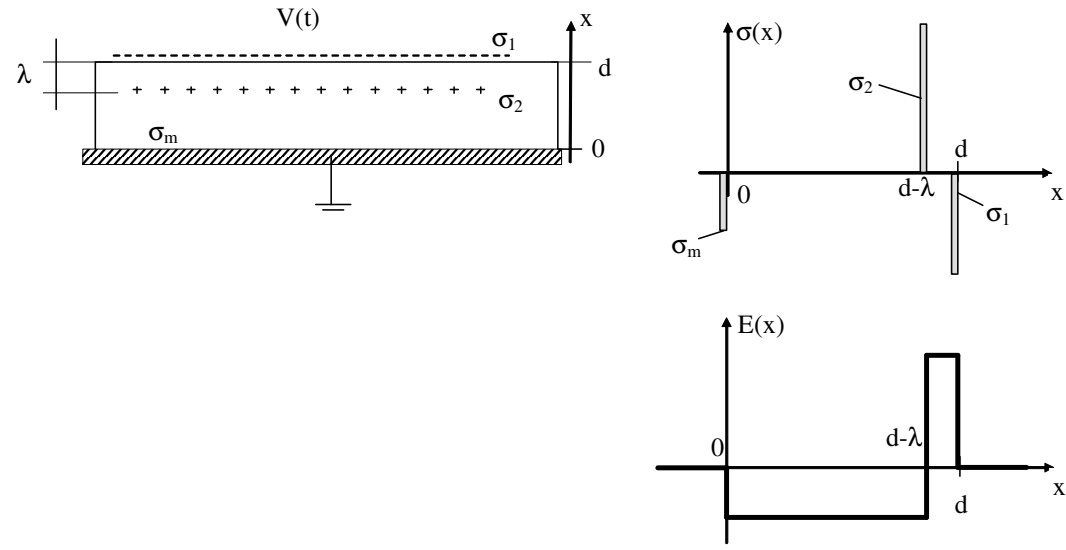

Figure 12. Distribution de charge dans le volume et à la surface avec représentation du champ électrique

Les champs à l'intérieur de l'isolant se déterminent alors par la relation de Poisson :

$$
\begin{aligned}
& \frac{d E}{d x}=\frac{\rho}{\varepsilon} \\
& E(d-\lambda<x<d)=-\frac{\sigma_{1}}{\varepsilon} \\
& E(0<x<d-\lambda)=-\frac{\sigma_{1}}{\varepsilon}-\frac{\sigma_{2}}{\varepsilon}
\end{aligned}
$$

On en déduit le potentiel de surface :

$$
V(t)=-\int_{0}^{d} E d l=\frac{1}{\varepsilon}\left(d \sigma_{1}+(d-\lambda) \sigma_{2}\right)
$$

Nous en déduisons :

$$
\frac{d V(t)}{d t}=\frac{1}{\varepsilon}\left(v_{1} \sigma_{1}+v_{2} \sigma_{2}\right)
$$

Avec, $v_{1}$ et $v_{2}$ les vitesses de déplacement des plans 1 et 2 . 
Les champs moyens, $E_{1}$ vu par le plan 1 et $E_{2}$ vu par le plan 2 sont :

$$
E_{1}=-\frac{\sigma_{1}}{2 \varepsilon} \quad \text { et } \quad E_{2}=-\frac{\sigma_{1}}{\varepsilon}-\frac{\sigma_{2}}{2 \varepsilon}
$$

$\mathrm{Si}$ on néglige le piégeage et si on suppose les mobilités de toutes les charges identiques et égales à $\mu$, en supposant par ailleurs les charges 1 négatives et 2 positives, on a alors : $v_{1}=-\mu E_{1}$ et $v_{2}=+\mu E_{2}$, ce qui entraîne :

$$
\frac{d V(t)}{d t}=-\frac{\mu}{2 \varepsilon^{2}}\left(-\sigma_{1}^{2}+\sigma_{2}\left(2 \sigma_{1}+\sigma_{2}\right)\right)
$$

$$
\text { Soit } \frac{d V(t)}{d t}=-\frac{\mu}{2 \varepsilon^{2}}\left(\sigma_{1}+\sigma_{2}-\sqrt{2} \sigma_{1}\right)\left(\sigma_{1}+\sigma_{2}+\sqrt{2} \sigma_{1}\right)
$$

Le premier terme entre parenthèses est nécessairement positif (puisque $\sigma_{1}$ est négatif et $\sigma_{1}+\sigma_{2}$ positif), en revanche le signe du second dépend des rapports entre $\sigma_{1}$ et $\sigma_{2}$.

On a un « retour » de potentiel si $\sigma_{1}+\sigma_{2}+\sqrt{2} \sigma_{1}$ est négatif, c'est à dire si

$$
\left|\sigma_{1}\right|>\frac{\sigma_{2}}{1+\sqrt{2}}
$$

Un tel modèle de double couche peut donc expliquer une montée initiale du potentiel. Le potentiel passera ensuite par un maximum, puis déclinera, ce qui ne peut s'expliquer par le modèle de «plans de charge » considéré ci-dessus, mais s'explique qualitativement simplement si on considère qu'un plan de champ nul traverse en réalité la distribution de charge 2 , de telle sorte que le mouvement de cette distribution sera la superposition du mouvement de retour vers la surface de sa partie supérieure, avec le mouvement de la partie inférieure de cette distribution qui continuera à s'injecter (Molinié, Alvarez 2005). Dans un premier temps le premier mouvement dominera, puis s'arrêtera lorsqu'elle aura rejoint la distribution de signe opposé. La deuxième contribution, celle d'injection, sera ensuite prédominante.

\subsection{Relaxation progressive de la pression électrostatique}

La pression électrostatique subie par le film étant proportionnelle au carré de la densité de charge de surface, supposer une irrégularité initiale de cette distribution de charge suivie d'une égalisation progressive, sous l'influence d'une conduction de surface, peut permettre d'expliquer une baisse de la pression électrostatique moyenne à densité moyenne de charge constante, et donc, une relaxation mécanique du film se traduisant par une hausse du potentiel.

On suppose que la charge est déposée avec une densité moyenne $\sigma_{0}$ sur la surface, mais qu'en réalité la charge est déposée sur une fraction $\alpha$ de cette surface, 
avec une densité $\frac{\sigma_{0}}{\alpha}$. Le module de la pression électrostatique vue par les zones chargées est alors donné par $P_{\text {initial }}=\frac{1}{2} \frac{\sigma_{0}{ }^{2}}{\alpha^{2} \varepsilon}$.

Après égalisation de la charge sur toute la surface, la pression électrostatique vue par les zones chargées devient $P_{\text {final }}=\frac{1}{2} \frac{\sigma_{0}{ }^{2}}{\varepsilon}$.

Le potentiel moyen de surface mesuré est proportionnel à la distance de la distribution de charge à la masse :

$$
\frac{V_{\text {final }}-V_{\text {initial }}}{V_{\text {initial }}}=\frac{d_{\text {final }}-d_{\text {initial }}}{d_{\text {initial }}}
$$

$\mathrm{Si}$ on suppose que la déformation de l'échantillon est proportionnelle à la pression moyenne, on a alors :

$$
\begin{aligned}
& \frac{V_{\text {final }}-V_{\text {initial }}}{V_{\text {initial }}}=\frac{P_{\text {initial }}-P_{\text {final }}}{\xi}, \xi \text { étant le module d'Young du matériau. } \\
& \text { C'est à dire }: \frac{V_{\text {final }}-V_{\text {initial }}}{V_{\text {initial }}}=\frac{1}{\xi} \frac{\sigma_{0}^{2}}{2 \varepsilon}\left(\frac{1}{\alpha^{2}}-1\right)=\frac{1}{\xi} \frac{\varepsilon V_{\text {final }}^{2}}{2 d_{\text {final }}^{2}}\left(\frac{1}{\alpha^{2}}-1\right)
\end{aligned}
$$

La remontée du potentiel dépend donc de l'hétérogénéité de la distribution de charge initiale et du rapport entre la pression électrostatique moyenne et le module d'Young de l'isolant.

\section{Discussion}

\subsection{Présence de plusieurs phénomènes superposés}

Deux études présentées précédemment dans le cadre de notre GdR (Agnel 2003, Jomni 2004) montrent l'existence de phénomènes de polarisation, ainsi qu'à partir d'une certaine valeur de champ une injection de la charge déposée.

Ces phénomènes contribuent à un déclin de potentiel, et sont une composante de nos résultats. Ainsi, il est très probable que la perte de potentiel très visible au centre des cartographies de la figure 11 soit liée à l'injection de charge. Ce phénomène, seulement visible dans le cas où le courant de la décharge corona est élevé, s'explique aisément par l'assistance fournie par l'énergie de la décharge à l'injection (Haridoss 1982). 


\subsection{Migration latérale des charges}

La migration latérale des charges est un des faits très nets présentés dans cette étude. Une telle migration a déjà été reportée plusieurs fois sur le PET dans le passé (par exemple Baum 1978). L'irrégularité de la migration superficielle dans notre cas s'explique en premier lieu par un état de surface mal défini. Par exemple, la tâche apparaissant sur la figure 10 est liée à une tâche sur la surface de l'échantillon, due au séchage d'une goutte d'eau déposée par inadvertance avant les essais.

Cependant nos observations montrent certains faits curieux, et qui se reproduisent d'une manière ou d'une autre dans tous nos essais, et qui tendent à montrer que ce n'est pas seulement le gradient latéral de charge ou la conductivité de la surface qui explique la migration. En effet, nous observons sur les cartographies différentielles comme sur la figure 11 le fait que l'augmentation du potentiel se développant en périphérie de la distribution n'est pas compensée par une baisse correspondante de potentiel dans une zone proche, mais au contraire dans une zone lointaine où le gradient de potentiel, pourtant, est dans une direction opposée. Il ne semble pas que cette observation soit un artefact, il est probable qu'elle ait un lien avec l'augmentation du potentiel lorsqu'on fait l'intégration sur l'ensemble de la surface.

Plus généralement ces cartographies nous paraissent délicates à expliquer en termes de simple conduction ou de migration de surface.

\subsection{Remontée du potentiel moyen}

Quel est le phénomène physique expliquant la remontée du potentiel moyen que nous observons sur les films?

Le modèle de double couche est il plausible ici ? L'hypothèse que dans le cas d'une charge corona avec faible distance pointe-grille, une certaine quantité d'ions de polarité opposée à celle de la pointe puisse être émise par le dispositif de charge, a déjà été formulée pour rendre compte de certains résultats expérimentaux (Dias 1989). Nous avons également envisagé la possibilité d'une instabilité dans notre circuit de charge qui puisse, par une oscillation du courant au moment de la coupure, produire une courte décharge sur la pointe de polarité opposée. Toutes ces hypothèses restent à confirmer expérimentalement. Par ailleurs, il semble peu probable qu'un modèle d'injection puisse expliquer nos résultats, car la présence de l'injection de charge devrait, comme dans le cas de la figure 11, se manifester plutôt par des points de faiblesse du potentiel dans le matériau, au centre de la distribution de charge. Or on observe plutôt cette remontée en périphérie. On peut donc penser que le modèle de double couche développé, intéressant du point de vue théorique, n'est pas adapté ici.

Le modèle de relaxation progressive de la pression électrostatique s'appuie sur le fait que le potentiel n'est mesuré qu'avec une résolution (500 $\mu \mathrm{m}$ environ) faible face à la finesse du matériau (23 $\mu \mathrm{m}$ ou $100 \mu \mathrm{m})$. Il est donc possible, qu'à une 
échelle proche de l'épaisseur du film, la charge déposée soit inhomogène, d'autant que les ions sont déposés à travers une grille (de pas environ 100 $\mu \mathrm{m})$. Des vérifications de cette inhomogénéité sont envisageables. On peut d'ores et déjà calculer la valeur du phénomène en supposant une valeur de $\alpha$ de l'ordre de 0,5 , et des valeurs de permittivité relative de l'ordre de 4 et du module d'Young de l'ordre de $10^{9} \mathrm{~Pa}$ (valeur mesurée sur le matériau que nous utilisons par l'équipe de D.Tréheux à Lyon). On obtient alors, pour une tension initiale de $2000 \mathrm{~V}$, une valeur de l'ordre de $0,1 \mathrm{~V}$ pour la remontée de potentiel. Pour obtenir une remontée de $10 \mathrm{~V}$, il faudrait supposer une valeur de $\alpha$ de l'ordre de 0,1 , ce qui n'est pas très réaliste. Par ailleurs le phénomène devrait être, pour une tension initiale donnée, 19 fois plus faible pour une épaisseur de $100 \mu \mathrm{m}$ que pour une épaisseur de $23 \mu \mathrm{m}$. Cela ne semble pas être le cas. En revanche, la présence de mécanismes piézoélectriques dans le PET pourrait conduire la pression électrostatique à engendrer des variations de potentiel plus importantes que le simple aplatissement du film que nous avons modélisé. Cette voie reste à explorer.

Nous pouvons aussi supposer qu'un mécanisme explique la remontée du potentiel, en déplaçant la charge électrique dans une direction opposée au champ. La diffusion de la charge sous l'effet d'un gradient de concentration est un des phénomènes envisageables a priori pour expliquer un tel mouvement. Néanmoins, nous ne voyons pas comment un gradient «inverse» de charge suffisamment important pourrait être produit dans le film pour pouvoir expliquer les effets observés. En revanche, sans que nous soyons en mesure de produire une véritable modélisation pour le moment, nous envisageons la possibilité que des forces physico-chimiques du type tension superficielle puissent amener la charge dans le matériau à migrer et à se regrouper suivant des lignes préférentielles. Cela pourrait éventuellement expliquer, à la fois la remontée globale du potentiel et les effets anormaux de migration de surface observés. Ce type de modélisation reste cependant à développer et à confronter à la littérature sur la physicochimie du PET.

\section{Conclusions}

L'observation répétée de phénomènes anormaux se traduisant par des remontées de potentiel dans nos essais nous a paru intéressante à reporter, même si nous ne sommes pas en mesure aujourd'hui d'en donner une explication inattaquable. Elle n'est pas attribuable à des artefacts, ni à des erreurs dans le mode opératoire. Elle est probablement liée à la combinaison spécifique de notre dispositif de charge et du matériau étudié (cet effet n'étant pas observé sur d'autres matériaux). L'hypothèse la plus probable que nous envisageons est aujourd'hui la présence de mécanismes physicochimiques permettant d'expliquer une migration de la charge (en volume ou en surface) contre le champ appliqué, ou des effets piézoélectriques dans le matériau. Un travail plus systématique est à entreprendre.

Il faut par ailleurs, plus généralement, souligner l'intérêt des cartographies de potentiel, concernant la détection de défauts ou les études de l'injection de charge 
sur des films à champ élevé : elles permettent de visualiser la dynamique de l'injection, à la fois spatialement et dans le temps, comme le montre la figure 11. Un tel outil nous paraît indispensable à la compréhension de ces phénomènes, et fournit enfin un moyen élégant de les visualiser.

\section{Références}

Agnel S. et al., «Étude du comportement électrique du PET par mesures de charges d'espace », Proc. Conf. EF'2003, 2003

Baum E. A., Lewis T. J., Toomer R., " The lateral motion of charge on thin films of polyethylene terephtalate», J. Phys. D., Vol. 11, pp. 963-977, 1978.

Coelho R., Jestin P., Levy L. and Sarrail D., «On the return-voltage buildup in insulating materials », IEEE Trans. Electr. Insul., Vol. 22, pp. 683-690, 1987.

Dias C. J., Marat-Mendes J. N., Giacometti J. A., «Effects of a corona discharge on the charge stability of Teflon FEP negative electrets », J. Phys. D: Appl. Phys., 22, 663-669, 1989.

Füzesséry S., «Polyéthylène téréphtalates (PET) pour films et feuilles », Techniques de l'Ingénieur, A 3375.

Haridoss S., Perlman M. M. and Carlone C., «Vibrationally excited diatomic molecules as charge injectors during corona charging of polymer films », J. Appl. Phys., Vol. 53, No. 9, pp. 6106-6114,1982.

Jomni F., Errhous L., El Kamel F., Sylvestre A., Molinié P., «The effect of the thermal ageing on the surface potential decay of polyethylene terephtalate », poster, $5^{\text {th }}$ International Conf. On Electric Charges in Non-Conductive Materials, Sfax, Novembre 2004.

Menguy C., «Electricité statique, principes, problèmes, applications », Techniques de l’Ingénieur, D 1035.

Molinié P., «Measuring and Modeling Transient Insulator Response to Charging: the Contribution of Surface Potential Studies », IEEE Tr. Diel. And El. Insulation, vol.12, pp. 939-950, 2005

Molinié P., Alvarez D., « Etude de l'injection de charge sur des plaques d'élastomère silicone par mesures du potentiel de surface », Conférence Matériaux pour le Génie Electrique, Lyon, décembre 2005.

Molinié P., Capron C., Brodin A. : " Apport des mesures de potentiel de surface à la caractérisation du vieillissement d'un revêtement anticorrosion ", Conférence Protection Corrosion Durabilité (PROCOD), Toulon, avril 2004

Notingher jr. P., Agnel S., Molinié P., Sylvestre A., Olariu M., Castellon J., Toureille A., Petre A., «Etude des charges d'espace dans le polyéthylène téréphtalate soumis a des forts champs électriques », Conférence Matériaux pour le Génie Electrique, Lyon, décembre 2005 . 